\title{
New 2-Benzoxazolinone Derivatives with Cytotoxic Activities from the Roots of Acanthus ilicifolius
}

\author{
Dan Zhao, ${ }^{a, b, \#}$ Lijun Xie, ${ }^{c, \#}$ Lei Yu, ${ }^{b}$ Ni An, ${ }^{a, b}$ Wei Na, ${ }^{a}$ Feng Chen, ${ }^{a}$ Youbin Li, ${ }^{a}$ \\ Yinfeng Tan, ${ }^{*, a}$ and Xiaopo Zhang*,a \\ ${ }^{a}$ School of Pharmaceutical Science, Hainan Medical University; Haikou 571199, P. R. China: ${ }^{b}$ Research Center on \\ Life Sciences and Environmental Sciences, Harbin University of Commerce; Harbin 150076, P. R. China: and ${ }^{c}$ Fujian \\ Institute of Microbiology; Fujian 350007, P.R. China. \\ Received July 29, 2015; accepted August 29, 2015
}

Four new 2-benzoxazolinone-type alkaloids (acanthosides A-D) along with three known ones were isolated from the roots of Acanthus ilicifolius. Their structures were established by detailed interpretation of one dimensional (1D)- and two dimensional (2D)-NMR as well as high-resolution electrospray ionization (ESI)MS data. The antiproliferative activities of these compounds were evaluated in vitro against three cultured cancer cell lines. The new compounds exhibited different levels of cytotoxicity against the HepG2, HeLa, and A-549 cancer cell lines with $\mathrm{IC}_{50}$ range 7.8-26.6 $\mu \mathrm{M}$. In comparison with known compounds, the new isolates displayed better cytotoxic activities, which was attributable to the presence of substituted benzoyl moiety in their structures.

Key words Acanthus ilicifolius; 2-benzoxazolinone; cytotoxic activity

The genus Acanthus belonging to the family Acanthaceae comprises over 30 species, many of which are spread in Africa, Asia, and other tropical as well as subtropical regions of the world. ${ }^{1)} A$. ilicifolius is a spiny herb found in the mangroves of southern China, Thailand, etc. As traditional medicine, this plant was used to treat various diseases such as tumor and hepatitis. ${ }^{2)}$ Previous investigations on the chemical constituents from the plant have led to the isolation of alkaloids, lignans and flavonoids. ${ }^{3,4)}$ The alkaloids obtained from this plant characterized with novel structures and significant biological activities have aroused interests from researchers worldwide. ${ }^{5)}$ Our discovery efforts searching for new antitumor agents from Hainan island region prompted us to focus on the mangrove plant A. ilicifolius. During our investigation, four new 2-benzoxazolinone-type alkaloids, named acanthosides A-D (1-4), were discovered from the roots of $\mathrm{A}$. ilicifolius. Meanwhile, their cytotoxic activities were evaluated in vitro. Herein, the isolation, and structural elucidation as well as in vitro cytotoxic evaluation of the new isolates against HepG2, HeLa, and A-549 cancer cell lines are described.

\section{Results and Discussion}

The air-dried and powdered roots of A. ilicifolius were extracted with 95\% ethanol under reflux. Following, the ethanol extract was filtered and concentrated under reduced pressure to yield a crude extract, which was suspended in distilled water and then successively partitioned with petroleum ether, and butanol. Phytochemical investigation on butyl alcohol $(\mathrm{BuOH})$ fraction has resulted in the isolation of four new 2-benzoxazolinone derivatives, named acanthosides A (1-4), and three known ones identified as 2-benzoxazolinone (5), 4-hydroxy-benzoxazolinone-2(3H)-one (6), 4- $O-\beta$-Dglucopyranosyl-benzoxazolin-2(3H)-one (7), respectively ${ }^{6,7)}$ (Fig. 1).

\footnotetext{
${ }^{\#}$ These authors contributed equally to this work.

* To whom correspondence should be addressed. e-mail: secondmessenger@163.com; z_xp1412@163.com
}

Acanthoside A (1) was isolated as colorless needle crystal, and determined to have a molecular formula of $\mathrm{C}_{22} \mathrm{H}_{23} \mathrm{NNaO}_{12}$ based on the high resolution-electrospray ionization (HRESI)-MS at $m / z 516.1109[\mathrm{M}+\mathrm{Na}]^{+}\left(\mathrm{Calcd} 516.1118[\mathrm{M}+\mathrm{Na}]^{+}\right)$. Its IR spectrum exhibited absorption bands due to hydroxy $\left(3450 \mathrm{~cm}^{-1}\right)$, carbonyl $\left(1760 \mathrm{~cm}^{-1}\right)$, and aromatic (1651, $1580 \mathrm{~cm}^{-1}$ ) functionalities. The ${ }^{1} \mathrm{H}-\mathrm{NMR}$ spectrum of $1 \mathrm{com}-$ prised five signals attributable to two aromatic rings resonated at $\delta_{\mathrm{H}} 7.37(2 \mathrm{H}, \mathrm{s}), 6.86(1 \mathrm{H}, \mathrm{dd}, 8.4,1.2 \mathrm{~Hz}), 7.00(1 \mathrm{H}$, t, $8.4 \mathrm{~Hz}), 6.92(1 \mathrm{H}, \mathrm{dd}, 8.4,1.2 \mathrm{~Hz})$. Moreover, the ${ }^{1} \mathrm{H}-\mathrm{NMR}$ spectrum displayed the presence of a glucosyl moiety with the anomeric proton signal at $\delta_{\mathrm{H}} 5.35(1 \mathrm{H}, \mathrm{d}, 7.8 \mathrm{~Hz})$ as well as the existence of two methoxy groups at $\delta_{\mathrm{H}} 3.87(6 \mathrm{H}, \mathrm{s})$. The ${ }^{13} \mathrm{C}-\mathrm{NMR}$ displayed typical signals at $\delta_{\mathrm{C}} 156.9,114.3,123.7$, $106.2,122.5,142.9,146.7,102.1,75.9,76.2,71.6,78.7,62.5$ assignable to a 4 - $O$ - $\beta$-D-glucopyranosyl-benzoxazolin- $2(3 H)$ one moiety. ${ }^{7)}$ The carbon signals resonated at $\delta_{\mathrm{C}} 167.8,121.2$, 108.7, 142.6, 57.1 together with signals at $\delta_{\mathrm{H}} 7.37(2 \mathrm{H}, \mathrm{s})$ were clearly ascribed to be a 3,5-dimethoxy-4-hydroxy-benzoyl moiety. Comparing the ${ }^{13} \mathrm{C}-\mathrm{NMR}$ spectral data of $\mathbf{1}$ with 4-O- $\beta$-D-glucopyranosyl-benzoxazolin-2(3H)-one revealed the downfield shift of C-2' $(+0.5 \mathrm{ppm})$ and upfield shifts of $\mathrm{C}-1^{\prime}$ and $\mathrm{C}-3^{\prime}$ ( -2.5 and $-2.1 \mathrm{ppm}$, respectively) of the glucosyl moiety indicating that the additional unit is an ester located at $\mathrm{C}-2^{\prime}{ }^{8)}$ Furthermore, the heteronuclear multiple bond connectivity (HMBC) spectrum revealed a correlation between $\mathrm{H}-2^{\prime}$ at $\delta_{\mathrm{H}} 5.22$ and C-7" at $\delta_{\mathrm{C}} 167.8$ as shown in Fig. 2. This clearly suggested the 3,5-dimethoxy-4-hydroxy-benzoyl moiety was connected with the glucosyl group through C-2' and C-7". Collectively, the chemical structure of $\mathbf{1}$ was established as shown in Fig. 1 with a given name acanthoside A.

Acanthoside B (2) was purified as colorless powder. Its molecular formula was determined to be the same as $\mathbf{1}$ by the HR-ESI-MS data. ${ }^{1} \mathrm{H}$ - and ${ }^{13} \mathrm{C}$-NMR data were superposable upon those of 1, apart from the attachment of 3,5-dimethoxy4-hydroxy-benzoyl moiety (C-7") to the glucosyl group (C-3'), 

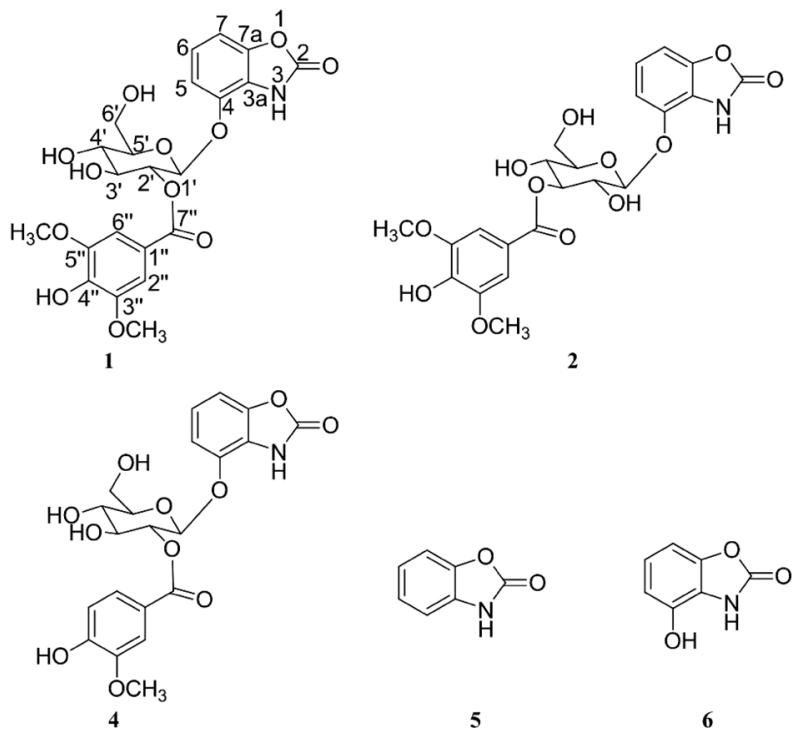

Fig. 1. Chemical Structures of Compounds 1-7<smiles>O=c1[nH]c2ccccc2o1</smiles><smiles>O=c1[nH]c2c(O)cccc2o1</smiles><smiles>O=c1[nH]c2c(OC3OC4OC(O3)C(O)C(O)C4O)cccc2o1</smiles>

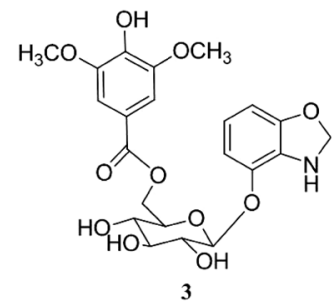

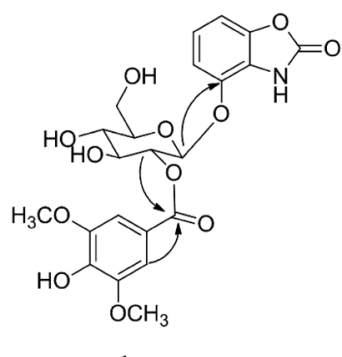

1

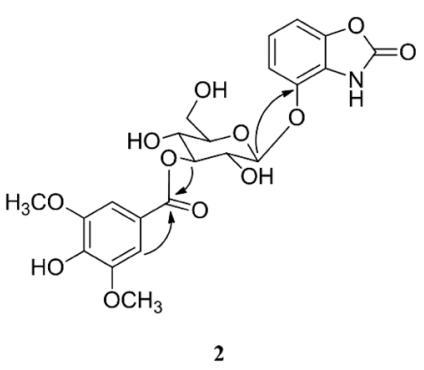

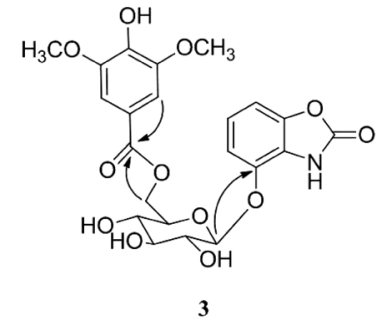

Fig. 2. Key HMBC Correlations $(\rightarrow)$ of Compounds 1-3

which was supported by HMBC correlations between $\mathrm{H}-3^{\prime}\left(\delta_{\mathrm{H}}\right.$ 5.28) and C-7" $\left(\delta_{\mathrm{C}}\right.$ 168.1) as shown in Fig. 2.

Acanthoside C (3), obtained as a colorless crystal, has the molecular formula $\mathrm{C}_{22} \mathrm{H}_{23} \mathrm{NO}_{12}$ as determined by HR-ESI-MS and NMR data. According to its molecular formula and NMR spectroscopic data, 3 was determined to have the same carbon framework as $\mathbf{1}$ and $\mathbf{2}$, differing only in that the attachment of 3,5-dimethoxy-4-hydroxy-benzoyl moiety to the glucosyl group was C-7" to C- 6 ', as confirmed by the HMBC cross peaks from $\mathrm{H}-6^{\prime}\left(\delta_{\mathrm{H}} 4.70,4.48\right)$ to $\mathrm{C}-7^{\prime \prime}\left(\delta_{\mathrm{C}} 167.9\right)$ as shown in Fig. 2.

Acanthoside D (4) was obtained as a mixture of 1 with an approximately ratio of the amounts of $3: 1$ by integration of certain clearly discernible paired protons in their ${ }^{1} \mathrm{H}-\mathrm{NMR}$ spectrum. However, the two compounds could not be completely separated by any chromatographic methods used in present study. Fortunately, their spectroscopic data were considered to be sufficient for the structural elucidation described below. The elemental formula of $\mathbf{4}$ was determined to be $\mathrm{C}_{21} \mathrm{H}_{21} \mathrm{NO}_{11}$ as deduced from the HR-ESI-MS data. The ${ }^{1} \mathrm{H}$ - and ${ }^{13} \mathrm{C}-\mathrm{NMR}$ spectral data were compatible with those of 1 except the 3,5-dimethoxy-4-hydroxy-benzoyl moiety was replaced by 3 -methoxy-4-hydroxy-benzoyl group in 4 . This was unambiguously confirmed by the observed ${ }^{13} \mathrm{C}-\mathrm{NMR}$ spectrum data and HMBC cross peak between $\mathrm{H}-2^{\prime}\left(\delta_{\mathrm{H}} 5.22\right)$ to $\mathrm{C}-7^{\prime \prime}\left(\delta_{\mathrm{C}} 167.8\right)$ as shown in Fig. 2.

The antiproliferative activities of the obtained compounds were evaluated in vitro against HepG2, HeLa, and A-549 cancer cell lines. They displayed different levels of cytotoxic activities with $\mathrm{IC}_{50}$ value range $7.8-26.6 \mu \mathrm{M}$. Previously, the extract of $A$. ilicifolius and 2-benzoxazolinone derivatives have been proved to possess anti-tumor effects. ${ }^{2,9)}$ In present research, the new isolates showed better antiproliferative activities than the three known ones, which might be affected by the existence of substituted benzoyl moiety in their structures. Additionally, the four new compounds showed activities differing in degrees, in which they exhibited most potent activities against HepG2. We highlight that these new structures with benzoyl groups linked to the alkaloid scaffold displays noticeable cytotoxic activities, providing scientific evidence for finding new therapeutic agents in treating tumor.

\section{Experimental}

General Experimental Procedures Optical rotations were measured on a Perkin-Elmer 241 polarimeter (Waltham, MA, U.S.A.), and UV data were recorded on Beckman Coulter DU 800 spectrometer (Tokyo, Japan). IR data were recorded using a Shimadzu FTIR-8400S spectrophotometer (Tokyo, Japan). NMR experiments were performed on Bruker AV III 600 spectrometers operating at $600 \mathrm{MHz}$ for ${ }^{1} \mathrm{H}$ and $150 \mathrm{MHz}$ for ${ }^{13} \mathrm{C}$, respectively. Chemical shifts are expressed in $\delta(\mathrm{ppm})$ referenced to solvent peaks at $\delta_{\mathrm{H}} 3.31$ and $\delta_{\mathrm{C}} 49.2$ for $\mathrm{CD}_{3} \mathrm{OD}$, and coupling constants are in Hz. HR-ESI-MS spectra were obtained from a Thermo Scientific LTQ-Obitrap 
Table 1. ${ }^{1} \mathrm{H}-$, and ${ }^{13} \mathrm{C}-\mathrm{NMR}$ Spectral Data for Acanthosides A-D (1-4) $\left(\mathrm{CD}_{3} \mathrm{OD}\right)$

\begin{tabular}{|c|c|c|c|c|c|c|c|c|}
\hline Position & & 1 & & 2 & & 3 & & 4 \\
\hline 2 & 156.9 & & 157.1 & & 157.0 & & 156.9 & \\
\hline 4 & 142.9 & & 143.3 & & 143.3 & & 142.2 & \\
\hline 5 & 114.3 & $6.86(1 \mathrm{H}, \mathrm{dd}, 8.4,1.2)$ & 114.7 & $7.11(1 \mathrm{H}, \mathrm{dd}, 8.4,1.2)$ & 113.7 & $6.97(1 \mathrm{H}, \mathrm{dd}, 8.4,1.2)$ & 114.2 & $6.85(1 \mathrm{H}, \mathrm{dd}, 8.4,1.2)$ \\
\hline 6 & 123.7 & $7.00(1 \mathrm{H}, \mathrm{t}, 8.4)$ & 123.8 & $7.01(1 \mathrm{H}, \mathrm{t}, 8.4)$ & 123.6 & $6.75(\mathrm{t}, 8.4)$ & 123.7 & $7.00(1 \mathrm{H}, \mathrm{t}, 8.4)$ \\
\hline 7 & 106.2 & $6.92(1 \mathrm{H}, \mathrm{dd}, 8.4,1.2)$ & 106.2 & $6.98(1 \mathrm{H}, \mathrm{dd}, 8.4,1.2)$ & 106.3 & $6.85(1 \mathrm{H}, \mathrm{dd}, 8.4,1.2)$ & 106.2 & $6.91(1 \mathrm{H}, \mathrm{dd}, 8.4,1.2)$ \\
\hline $7 \mathrm{a}$ & 146.7 & & 146.7 & & 146.4 & & 146.7 & \\
\hline $3 a$ & 122.5 & & 122.8 & & 122.6 & & 122.6 & \\
\hline $1^{\prime}$ & 102.1 & $5.35(1 \mathrm{H}, \mathrm{d}, 7.8)$ & 104.2 & $5.06(1 \mathrm{H}, \mathrm{d}, 7.8)$ & 103.7 & $4.95(1 \mathrm{H}, \mathrm{d}, 7.8)$ & 102.0 & $5.32(1 \mathrm{H}, \mathrm{d}, 7.8)$ \\
\hline $2^{\prime}$ & 75.9 & $5.22(1 \mathrm{H}, \mathrm{dd}, 9.6,7.8)$ & 73.6 & $3.80(1 \mathrm{H}, \mathrm{dd}, 9.6,7.8)$ & 75.1 & $3.65(1 \mathrm{H}, \mathrm{m})$ & 75.7 & $5.22(1 \mathrm{H}, \mathrm{dd}, 9.6,7.8)$ \\
\hline $3^{\prime}$ & 76.2 & $3.81(1 \mathrm{H}, \mathrm{t}, 9.6)$ & 79.4 & $5.28(1 \mathrm{H}, \mathrm{t}, 9.6)$ & 78.0 & $3.80(1 \mathrm{H}, \mathrm{td}, 7.8,2.4)$ & 76.3 & $3.80(1 \mathrm{H}, \mathrm{t}, 9.6)$ \\
\hline $4^{\prime}$ & 71.6 & $3.56(1 \mathrm{H}, \mathrm{m})$ & 69.7 & $3.58(1 \mathrm{H}, \mathrm{m})$ & 72.2 & $3.45(1 \mathrm{H}, \mathrm{t}, 7.8)$ & 71.6 & $3.55(1 \mathrm{H}, \mathrm{m})$ \\
\hline $5^{\prime}$ & 78.7 & $3.57(1 \mathrm{H}, \mathrm{m})$ & 78.4 & $3.60(1 \mathrm{H}, \mathrm{m})$ & 76.1 & $3.53(1 \mathrm{H}, \mathrm{m})$ & 78.6 & $3.56(1 \mathrm{H}, \mathrm{m})$ \\
\hline $6 a$ & 62.5 & $3.95(1 \mathrm{H}, \mathrm{dd}, 12.0,1.8)$ & 62.3 & $3.95(1 \mathrm{H}, \mathrm{dd}, 12.0,2.4)$ & 65.1 & $4.70(1 \mathrm{H}, \mathrm{dd}, 12.0,2.4)$ & 62.5 & $3.94(1 \mathrm{H}, \mathrm{dd}, 12.0,1.8)$ \\
\hline $6 \mathrm{~b}$ & & $3.77(1 \mathrm{H}, \mathrm{dd}, 12.0,5.4)$ & & $3.78(1 \mathrm{H}, \mathrm{dd}, 12.0,4.8)$ & & $4.48(1 \mathrm{H}, \mathrm{dd}, 12.0,4.8)$ & & $3.76(1 \mathrm{H}, \mathrm{dd}, 12.0,5.4)$ \\
\hline $1^{\prime \prime}$ & 121.2 & & 121.2 & & 121.4 & & 122.4 & \\
\hline $2^{\prime \prime}$ & 108.7 & $7.37(1 \mathrm{H}, \mathrm{s})$ & 108.6 & $7.42(1 \mathrm{H}, \mathrm{s})$ & 108.6 & $7.30(1 \mathrm{H}, \mathrm{s})$ & 116.2 & $7.59(1 \mathrm{H}, \mathrm{d}, 1.8)$ \\
\hline $3^{\prime \prime}$ & 149.2 & & 149.1 & & 149.1 & & 148.9 & \\
\hline $4^{\prime \prime}$ & 142.6 & & 142.3 & & 142.4 & & 153.4 & \\
\hline $5^{\prime \prime}$ & 149.2 & & 149.1 & & 149.1 & & 114.0 & $6.86(1 \mathrm{H}, \mathrm{d}, 8.4)$ \\
\hline $6^{\prime \prime}$ & 108.7 & $7.37(1 \mathrm{H}, \mathrm{s})$ & 108.6 & $7.42(1 \mathrm{H}, \mathrm{s})$ & 108.6 & $7.30(1 \mathrm{H}, \mathrm{s})$ & 125.6 & $7.62(1 \mathrm{H}, \mathrm{dd}, 8.4,1.8)$ \\
\hline$-\mathrm{COO}$ & 167.8 & & 168.1 & & 167.9 & & 167.8 & \\
\hline$-\mathrm{OCH}_{3}$ & 57.1 & $3.87(6 \mathrm{H}, \mathrm{s})$ & 57.1 & $3.91(6 \mathrm{H}, \mathrm{s})$ & 57.1 & $3.85(6 \mathrm{H}, \mathrm{s})$ & 56.6 & $3.88(3 \mathrm{H}, \mathrm{s})$ \\
\hline
\end{tabular}

XL instrument (Thermo Scientific, Bremen, Germany). Silica gel (200-300 mesh, Qingdao Marine Chemistry Co., Ltd.) and Sephadex LH-20 (GE Healthcare, U.S.A.) were used for column chromatography. HPLC chromatography was performed on LC-6AD equipped with a UV detector of SPD-10A (Shimadzu, Kyoto, Japan), and a column of ZORAX SB-phenyl ( $250 \times 10 \mathrm{~mm}, 5 \mu \mathrm{m}$, Agilent Technologies Co., Ltd., U.S.A.).

Sample Collection The roots of A. ilicifolius were collected from Wenchang County, Hainan Province of People's Republic of China in July 2014. The sample was identified by Dr. Niankai Zeng and the voucher specimen (No. A1201407) has been deposited in the Herbarium of School of Pharmaceutical Science, Hainan Medical University.

Extraction and Isolation The air-dried roots of $A$. ilicifolius $(2.0 \mathrm{~kg})$ were cut into piece and extracted with $95 \% \mathrm{EtOH}$ $(3 \times 8.0 \mathrm{~L})$ at $70^{\circ} \mathrm{C}$ for $2 \mathrm{~h} / 2$ times. The ethanol extract was concentrated under reduced pressure at room temperature. After evaporation of the solvent, the residue was suspended in water and extracted with petroleum ether, and $n-\mathrm{BuOH}$, successively. The $n$-BuOH parts $(20 \mathrm{~g})$ was subject to silica gel column chromatography (CC) $(45 \times 12 \mathrm{~cm}, 200-300$ mesh, $200 \mathrm{~g})$, using chloroform-acetone gradient elution $(9: 1,6: 1,4: 1,2: 1,1: 1)$ to afford six fractions (Frs. A-F). Fr. A $(9.8 \mathrm{~g})$ was purified by semi-preparative HPLC $\left(45 \% \mathrm{MeOH}\right.$ in $\mathrm{H}_{2} \mathrm{O}, 2.0 \mathrm{~mL} / \mathrm{min}$, $210 \mathrm{~nm})$ to afford $\left.5\left(t_{\mathrm{R}} 22.4 \mathrm{~min}, 20.6 \mathrm{mg}\right)\right)$ and $\mathbf{6}\left(t_{\mathrm{R}} 18.2 \mathrm{~min}\right.$, $5.0 \mathrm{mg})$. Fr. B ( $4.0 \mathrm{~g})$ was chromatographed over Sephadex $\mathrm{LH}-20$ using $\mathrm{MeOH}$ as eluent, to give ten fractions (B1-10). The second fraction $(\mathrm{B} 2,25 \mathrm{mg})$ was purified by semi-preparative $\mathrm{HPLC}\left(35 \% \mathrm{MeOH}\right.$ in $\left.\mathrm{H}_{2} \mathrm{O}, 2.0 \mathrm{~mL} / \mathrm{min}, 210 \mathrm{~nm}\right)$ to afford $1\left(t_{\mathrm{R}} 16.0 \mathrm{~min}, 3.5 \mathrm{mg}\right)$. Fr. D $(3.5 \mathrm{~g})$ was subjected to Sephadex $\mathrm{LH}-20$ eluted with $\mathrm{MeOH}$ to give nine fractions (E1-9), the second fraction $(\mathrm{E} 2,35 \mathrm{mg})$ was further purified by semipreparative $\mathrm{HPLC}\left(45 \% \mathrm{MeOH}\right.$ in $\left.\mathrm{H}_{2} \mathrm{O}, 2.0 \mathrm{~mL} / \mathrm{min}, 210 \mathrm{~nm}\right)$ to afford $2\left(t_{\mathrm{R}} 15.6 \mathrm{~min}, 2.3 \mathrm{mg}\right)$ and $\mathbf{3}\left(t_{\mathrm{R}} 26.0 \mathrm{~min}, 2.8 \mathrm{mg}\right) .4$ $(2.1 \mathrm{mg}$ ) was obtained as a mixture with $\mathbf{1}$ by semi-preparative
Table 2. Growth Inhibition of 1-7 against Three Cancer Cell Lines

\begin{tabular}{crcc}
\hline \hline \multirow{2}{*}{ Compound } & \multicolumn{3}{c}{ Cytotoxicity $\left(\mathrm{IC}_{50}\right.$ in $\left.\mu \mathrm{M}\right)$} \\
\cline { 2 - 4 } & HepG2 & HeLa & A549 \\
\hline $\mathbf{1}$ & $7.8 \pm 2.8$ & $18.4 \pm 5.8$ & $12.4 \pm 5.6$ \\
$\mathbf{2}$ & $9.6 \pm 3.4$ & $25.8 \pm 7.6$ & $11.5 \pm 5.2$ \\
$\mathbf{3}$ & $12.8 \pm 5.2$ & $26.6 \pm 8.4$ & $12.8 \pm 5.8$ \\
$\mathbf{4}$ & $12.4 \pm 3.6$ & $16.8 \pm 4.8$ & $17.4 \pm 8.6$ \\
$\mathbf{5}$ & $27.8 \pm 8.0$ & - & $16.8 \pm 7.6$ \\
$\mathbf{6}$ & $24.4 \pm 7.8$ & - & $15.6 \pm 8.4$ \\
$\mathbf{7}$ & $15.6 \pm 8.0$ & $20.2 \pm 6.8$ & $18.2 \pm 8.8$ \\
Doxorubicin & $1.32 \pm 0.03$ & $0.58 \pm 0.14$ & $0.76 \pm 0.24$ \\
\hline
\end{tabular}

HPLC (45\% MeOH in $\left.\mathrm{H}_{2} \mathrm{O}, t_{\mathrm{R}} 17.5 \mathrm{~min}\right)$ from the third fraction (E3, 20 mg) since the two compounds could not be completely separated by any chromatographic methods used in present study. Fr. E (6.5 g) was subjected to Sephadex LH-20 eluted with $\mathrm{MeOH}$ to give nine fractions (F1-9). F4 (135.0 mg) was purified by semi-preparative HPLC $\left(25 \% \mathrm{MeOH}\right.$ in $\mathrm{H}_{2} \mathrm{O}$, $2.0 \mathrm{~mL} / \mathrm{min}, 210 \mathrm{~nm})$ to afford $7\left(t_{\mathrm{R}} 26.2 \mathrm{~min}, 11.3 \mathrm{mg}\right)$.

Acanthoside A (1): Colorless needle crystal; ${ }^{1} \mathrm{H}-$ and ${ }^{13} \mathrm{C}-\mathrm{NMR}\left(\mathrm{CD}_{3} \mathrm{OD}\right)$ data: see Table $1 .[\alpha]_{\mathrm{D}}^{25}-28.5$ (c 0.067 , $\mathrm{MeOH})$; UV (MeOH) $\lambda_{\max } 224,275 \mathrm{~nm}$; IR (KBr) $v_{\max }$ : 3422, 1760, 1651, 1580, $1508 \mathrm{~cm}^{-1}$; HR-ESI-MS m/z: 516.1109 $[\mathrm{M}+\mathrm{Na}]^{+}$(Calcd 516.1118 for $\mathrm{C}_{22} \mathrm{H}_{23} \mathrm{NNaO}_{12}$ ).

Acanthoside B (2): Colorless powder; ${ }^{1} \mathrm{H}$ - and ${ }^{13} \mathrm{C}-\mathrm{NMR}$ $\left(\mathrm{CD}_{3} \mathrm{OD}\right)$ data: see Table 1. $[\alpha]_{\mathrm{D}}^{25}-7.5$ (c $\left.0.067, \mathrm{MeOH}\right)$; UV $(\mathrm{MeOH}) \lambda_{\max } 224,276 \mathrm{~nm}$; IR (KBr) $v_{\max }: 3420,1762,1650$, 1580, $1502 \mathrm{~cm}^{-1}$; HR-ESI-MS $m / z: 516.1114[\mathrm{M}+\mathrm{Na}]^{+}$(Calcd 516.1118 for $\mathrm{C}_{22} \mathrm{H}_{23} \mathrm{NNaO}_{12}$ ).

Acanthoside $\mathrm{C}(3)$ : Amorphous powder; ${ }^{1} \mathrm{H}$ - and ${ }^{13} \mathrm{C}-\mathrm{NMR}$ $\left(\mathrm{CD}_{3} \mathrm{OD}\right)$ data: see Table 1. $[\alpha]_{\mathrm{D}}^{25}-40.5$ (c 0.067, MeOH); UV $(\mathrm{MeOH}) \lambda_{\max } 224,276 \mathrm{~nm}$; IR (KBr) $v_{\text {max }}: 3423,1760,1652$, 1622, 1576, $1506 \mathrm{~cm}^{-1}$; HR-ESI-MS m/z: $516.1108[\mathrm{M}+\mathrm{Na}]^{+}$ 
(Calcd 516.1118 for $\mathrm{C}_{22} \mathrm{H}_{23} \mathrm{NNaO}_{12}$ ).

Acanthoside D (4): Amorphous powder; ${ }^{1} \mathrm{H}$ - and ${ }^{13} \mathrm{C}-\mathrm{NMR}$ $\left(\mathrm{CD}_{3} \mathrm{OD}\right)$ data: see Table 1. UV (MeOH) $\lambda_{\max } 225,287 \mathrm{~nm}$; IR (KBr) $v_{\max }: 3422,1761,1652,1620,1575,1508 \mathrm{~cm}^{-1}$; HR-ESI-MS $m / z$ : $486.1006[\mathrm{M}+\mathrm{Na}]^{+}$(Calcd 486.1012 for $\mathrm{C}_{21} \mathrm{H}_{21} \mathrm{NNaO}_{11}$ ).

Cytotoxicity Bioassays The following human cancer cell lines were used: HepG2, A-549, and HeLa. The cytotoxicity assay was performed using the 3-(4,5-dimethylthiazol2-yl)-2,5-diphenyltetrazolium bromide (MTT) method in 96well microplates. Half maximal inhibitory $\left(\mathrm{IC}_{50}\right)$ values were calculated by the previous method ${ }^{10)}$ (Table 2).

Acknowledgments We gratefully acknowledge financial support from the National Natural Science Foundation of China (No. 81560696, 81202994), and Natural Science Foundation of Hainan Province (No. 20158362).

Conflict of Interests The authors declare no conflict of interest.

\section{References}

1) Wu J., Zhang S., Xiao Q., Li Q., Huang J., Long L., Huang L., Phytochemistry, 63, 491-495 (2003).

2) Babu B. H., Shylesh B. S., Padikkala J., J. Ethnopharmacol., 79, 27-33 (2002).

3) Wu J., Zhang S., Huang J., Xiao Q., Li Q., Long L., Huang L., Chem. Pharm. Bull., 51, 1201-1203 (2003).

4) Kanchanapoom T., Kamel M. S., Kasai R., Picheansoonthon C., Hiraga Y., Yamasaki K., Phytochemistry, 58, 637-640 (2001).

5) Li M. Y., Xiao Q., Pan J. Y., Wu J., Nat. Prod. Rep., 26, 281-298 (2009).

6) Chatterjee A., Sharma N. J., Banerji J., Basa S. C., Indian J. Chem., 29B, 132 (1990).

7) Huo C., An D., Wang B., Zhao Y., Lin W., Magn. Reson. Chem., 43, 343-345 (2005).

8) Kanchanapoom T., Kamel M. S., Kasai R., Yamasaki K., Picheansoonthon C., Hiraga Y., Phytochemistry, 56, 369-372 (2001).

9) Bravo H. R., Copaja S. V., Figueroa-Duarte S., Lamborot M., San Martín J., Z. Naturforsch. C, 60, 389-393 (2005).

10) Zhang X., Tan Y., Li Y., Jin L., Wei N., Wu H., Ma G., Zheng Q., Tian Y., Yang J., Zhang J., Xu D., Chem. Pharm. Bull., 62, 494-498 (2014). 